\title{
Peak Oxygen Uptake and Mortality in Cystic Fibrosis: Systematic Review and Meta-Analysis
}

\author{
Fernanda Maria Vendrusculo, João Paulo Heinzmann-Filho, Juliana Severo da Silva, \\ Margarita Perez Ruiz, and Márcio Vinícius Fagundes Donadio
}

\begin{abstract}
BACKGROUND: Aerobic fitness, as measured by peak oxygen uptake ( $\dot{\mathrm{V}}_{\mathrm{O}_{2}}$ peak), correlates with survival in children and adults with cystic fibrosis $(\mathrm{CF})$. We sought to evaluate the effects of $\dot{\mathrm{V}}_{\mathrm{O}_{2}}$ peak on mortality rates in subjects with CF. METHODS: An online search in PubMed, Embase, LILACS, and SciELO databases was conducted, and cohort studies that assessed mortality rates after oxygen absorption measurements during a maximal exercise test were included. Data were extracted independently by 2 reviewers. The quality analysis of the selected articles was performed using the Newcastle-Ottawa scale. The main outcome evaluated was the mortality of subjects with CF. Whenever possible, and if appropriate, a random effect meta-analysis was performed. RESULTS: Six cohort studies were included in this systematic review including 551 subjects. Five studies were classified with high methodological quality. Two analyses were carried out to evaluate the influence of $\dot{\mathrm{V}}_{\mathrm{O}_{2}}$ peak on mortality. Total difference standardized mean between $\dot{\mathrm{V}}_{\mathrm{O}_{2}}$ peak averages in the survival or non-survival groups was $-0.606(95 \% \mathrm{CI}=-0.993$ to $-0.219, P=.002)$. In addition, subjects with a lower $\dot{\mathrm{V}}_{\mathrm{O}_{2}}$ peak had a significantly higher mortality risk (relative risk 4.896, $95 \% \mathrm{CI}=1.086$ to $22.072, P=.039)$ in an 8-y follow-up period. CONCLUSION: Low levels of peak oxygen uptake are associated with an increase of 4.9 in the risk of mortality in subjects with $\mathrm{CF}$. This indicates that $\dot{\mathbf{V}}_{\mathrm{O}_{2}}$ could be an important follow-up variable to measure, in addition to $F E V_{1}$. Key words: cystic fibrosis; oxygen consumption; exercise capacity; exercise test; mortality; survival. [Respir Care 2019;64(1):91-98. (c) 2019 Daedalus Enterprises]
\end{abstract}

\section{Introduction}

Cystic fibrosis (CF) is a multisystemic genetic disease characterized by progressive loss of lung function and consequent limitation of aerobic fitness. ${ }^{1}$ Although morbidity and mortality are still a major concern in the disease man-

\footnotetext{
Drs Heinzmann-Filho, Donadio, Vendrusculo, and Ms da Silva, are affiliated with the Laboratory of Pediatric Physical Activity, Centro Infant, Institute of Biomedical Research, Pontifícia Universidade Católica do Rio Grande do Sul, Porto Alegre, Rio Grande do Sul, Brazil. Dr Perez Ruiz is affiliated with the School of Doctoral Studies and Research, Universidad Europea de Madrid, Madrid, Spain.
}

The authors have disclosed no conflicts of interest.

Correspondence: Márcio Donadio, Laboratory of Pediatric Physical Activity, Centro Infant, Institute of Biomedical Research, Av. Ipiranga, 6690, $2^{\circ}$ andar, Porto Alegre, Rio Grande do Sul, CEP 90610-000, Brazil. E-mail: mdonadio@pucrs.br

DOI: $10.4187 /$ respcare. 06185 agement, life expectancy is increasing each year with advancement in the diagnosis and treatment; according to data from the Cystic Fibrosis Foundation, the expected median survival in the year 2015 was $41.7 \mathrm{y}^{2}$ Thus, as lung function is better maintained and aging of patients increases, other comorbidities rise and there is a growing importance of factors such as the preservation of aerobic fitness status. ${ }^{3}$

In general, many factors have been associated with a worse prognosis in individuals with $\mathrm{CF}$, among them sex, ${ }^{4}$ decline in lung function, ${ }^{4-7}$ number of exacerbations, ${ }^{6,7}$ nutritional status, ${ }^{7-9}$ chronic colonization of the airways, ${ }^{10,11}$ and peak oxygen consumption $\left(\dot{\mathrm{V}}_{\mathrm{O}_{2}}\right.$ peak)..$^{5,11,12} \mathrm{It}$ is known that the reduction of $\dot{\mathrm{V}}_{\mathrm{O}_{2}}$ peak is of multifactorial origin, given that the mechanisms that can limit aerobic fitness include respiratory, cardiovascular, and of peripheral muscles. ${ }^{13}$ Therefore, performance on the cardiopulmonary exercise test (CPET) depends on more than lung function and thus reflects the effects of the disease on other organ systems earlier on, ${ }^{14}$ although $\mathrm{FEV}_{1}$ has still been cited as 
the best predictor of mortality in children and adults with CF.4,11,12 Thus, considering only lung function as a disease progression marker may not identify other factors that lead to morbidity and mortality in these subjects. Therefore, aerobic fitness evaluation through CPET seems to be a more comprehensive method. ${ }^{15}$

CPET is considered the gold standard for evaluating aerobic fitness and has been used not only to follow the evolution of the disease, ${ }^{16}$ because it evaluates the performance and interaction of the cardiovascular, respiratory, and metabolic systems, ${ }^{17}$ but also for the prescription of physical exercise. ${ }^{18}$ In addition, studies have shown high reproducibility of CPET for young ${ }^{19}$ and adult ${ }^{20}$ subjects with $\mathrm{CF}$, demonstrating a coefficient of variation of $9.3 \%$ and $6.9 \%$, respectively. Moreover, the recent statement on exercise testing in CF recommends performing CPET in individuals aged $\geq 10 \mathrm{y}$ as an important part of the annual review process. ${ }^{15}$

Exercise intolerance due to hyperinflation and increased respiratory work caused by airway obstruction may be present as the disease advances, ${ }^{21}$ although it is increasingly common to find some pediatric patients who do not present dynamic hyperinflation during exercise. Aerobic fitness correlates with survival in children ${ }^{12}$ and adults 5,11 with CF. Nixon et al ${ }^{11}$ was the first to demonstrate that $\dot{\mathrm{V}}_{\mathrm{O}_{2}}$ peak ( $<45 \mathrm{~mL} / \mathrm{kg} / \mathrm{min}$ ) could be a predictor of survival in young individuals. A few years later, this finding was replicated by the study of Pianosi et al, ${ }^{12}$ which showed that subjects with $\dot{\mathrm{V}}_{\mathrm{O}_{2}}$ peak $>82 \%$ of predicted had a better rate of survival.

Thus, we hypothesized that a low $\dot{\mathrm{V}}_{\mathrm{O}_{2}}$ peak was an independent predictor of mortality in individuals with CF. Therefore, we performed a systematic review and quantitative meta-analysis of all available studies that reported the effects of peak oxygen consumption on mortality rates in individuals with $\mathrm{CF}$.

\section{Methods}

This systematic review was performed following the Meta-analysis of Observational Studies in Epidemiology (MOOSE) guidelines, ${ }^{22}$ and data were described according to the Preferred Reporting Items for Systematic Reviews and Meta-Analyses guidelines. ${ }^{23}$ The protocol of this review was registered in the international prospective register of systematic reviews and was accepted under the number CRD42016045759.

\section{Search Strategy}

Studies were identified from the following databases: PubMed, Embase, LILACS, and SciELO. The terms used were ("exercise test" or "exercise testing" or "cardiopulmonary exercise test" or "cardiopulmonary exercise test- ing" or "peak oxygen uptake" or "maximal oxygen consumption" or "exercise tolerance" or "exercise capacity") and ("mortality" or "survival") and ("cystic fibrosis"). No filters were used. A manual search was performed on the bibliographic references of the selected articles to search for additional publications that were pertinent to the study purpose. The searches were conducted in March 2017.

\section{Study Selection}

Two authors (FMV and JSdS) independently evaluated article titles and abstracts, thereby selecting the studies to be included in the review. Cohort studies of subjects with $\mathrm{CF}$ that assessed mortality rates after oxygen uptake measurements during a maximal exercise test were included. Articles that did not use variables of the maximal exercise test to predict mortality, articles that assessed the mortality of individuals already on the lung transplant list (because the inclusion of severely ill individuals would potentially bias the analysis), review studies, case studies, experimental models, abstracts, response letters, editorials, and duplicate publications, as well as those that did not meet inclusion criteria, were excluded after the analysis of the abstract or full text. Disagreements over the inclusion criteria of a particular study were discussed and resolved through consensus. A third author (MVFD) evaluated and resolved the discrepancies whenever disagreement or doubts between the 2 authors remained.

\section{Data Extraction}

The following data were extracted from each of the selected studies: title, first author's name, year of publication, country of origin, language of publication, type of study, age of subjects, sample size, type of equipment used, type of protocol used, duration of follow-up, $\dot{\mathrm{V}}_{\mathrm{O}_{2}}$ peak, breathing reserve, ventilatory equivalent for peak oxygen consumption $\left(\dot{\mathrm{V}}_{\mathrm{E}} / \dot{\mathrm{V}}_{\mathrm{O}_{2}}\right)$, and ventilatory equivalent for carbon dioxide production $\left(\dot{\mathrm{V}}_{\mathrm{E}} / \mathrm{V}_{\mathrm{CO}_{2}}\right)$. In addition, data from the variables of the cardiopulmonary exercise test and other variables that could predict the mortality of $\mathrm{CF}$ patients were collected. Any discrepancies were resolved by consensus among the researchers. Moreover, whenever appropriate, an attempt to contact authors of the included studies was performed in order to request additional information.

\section{Quality Analysis}

The quality analysis of the selected articles was performed individually by 2 authors (FMV and JPH-F) using the Newcastle-Ottawa Scale, which assigns a maximum of 9 points to the highest quality studies according to the 3 evaluated parameters: selection (4 points), comparability 
( 2 points), and outcome ( 3 points). ${ }^{24}$ The overall quality of the study was defined as poor (score $0-3$ ), reasonable (score 4-6), or high (score 7-9). The findings were discussed together with a third author (MVFD), and the discrepancies or doubts were resolved by consensus.

\section{Synthesis of Data and Statistical Analysis}

When possible and appropriate, a randomized metaanalysis of the studies was conducted. The standard difference of the $\dot{\mathrm{V}}_{\mathrm{O}_{2}}$ peak averages between the survival and non-survival groups was performed by extracting the average data, sample size, and $P$ value from each study included in that analysis. The association of high or low $\dot{\mathrm{V}}_{\mathrm{O}_{2}}$ peak with mortality was calculated between the survival and non-survival groups by extracting the relative risk and the $95 \% \mathrm{CI}$ and/or the number of events (death) and the total of participants in each group, using the DerSimonianLaird randomized model.

Forest plots with the point size reflecting study weight were used to graphically represent the results of metaanalysis. The $\mathrm{I}^{2}$ and $\mathrm{Q}$ tests were used to quantify the degree of heterogeneity between the studies. It was not possible to evaluate the bias of publication with the Egger's test due to the small number of studies included in each analysis. All analysis were performed in the comprehensive meta-analysis software (Biostat, Englewood, New Jersey).

\section{Results}

A total of 8,698 articles were found (3,886 in PubMed, 4,787 in EMBASE, and 25 in LILACS). Of these, 2,786 studies were excluded because they were repeated in the database searches, and 5,904 studies were excluded because they did not meet the eligibility criteria of our study. Eight studies were assessed for eligibility, and after analysis of the full text, 2 studies were excluded for including individuals already on a transplant list. ${ }^{25,26}$ Therefore, 6 studies were included in this review, and 4 studies were included in the meta-analysis. Figure 1 shows the flow chart of the articles found and the reasons for the exclusion of studies.

The selected articles included a total of 551 subjects, and the sample size of each study varied between 28 and 149 subjects, with ages ranging from 10 to $30.2 \mathrm{y}$ and follow-up time between 2.8 and 8 y (Table 1). Only 2 studies presented CFTR genotype classification, although no separate analyses were performed.9,10 All studies used the cycle ergometer to perform CPET. However, when evaluating the type of protocol, 3 studies used the Godfrey protocol, ${ }^{9-11} 2$ performed a ramp protocol, ${ }^{5,27}$ and 1 study ${ }^{12}$ used a step increment. The $\dot{\mathrm{V}}_{\mathrm{O}_{2}}$ peak, breathing reserve,

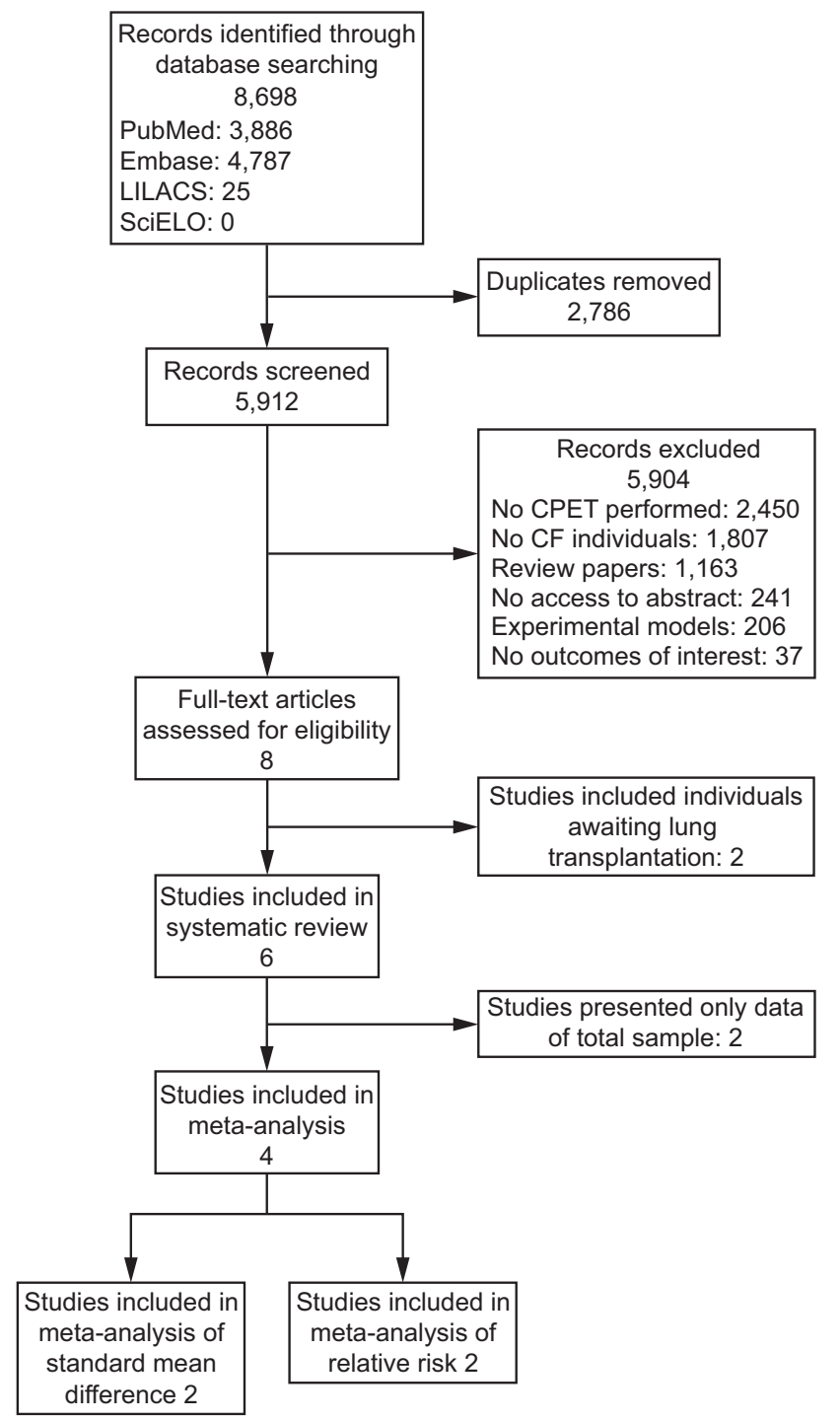

Fig. 1. Flow chart. CPET = cardiopulmonary exercise testing; $\mathrm{CF}=$ cystic fibrosis.

$\dot{\mathrm{V}}_{\mathrm{E}} / \dot{\mathrm{V}}_{\mathrm{O}_{2}}$, and $\dot{\mathrm{V}}_{\mathrm{E}} / \dot{\mathrm{V}}_{\mathrm{CO}_{2}}$ data from each article are presented in Table 2.

Regarding the overall methodological quality, 5 of the included studies $5,9,11,12,27$ were classified as high quality, and two $\mathrm{o}^{5,11}$ of these received the maximum score (9 points). On the other hand, only 1 article $^{10}$ was classified as reasonable quality and was assigned 6 points. Table 3 presents the parameters evaluated in the Newcastle-Ottawa Scale.

Table 4 shows the data of significant variables to predict mortality in CF subjects. $\dot{\mathrm{V}}_{\mathrm{O}_{2}}$ peak was significant in 5 studies,, $59-12$ and breathing reserve was significant in 1 study. ${ }^{9}$ In the study by Nguyen et al, ${ }^{27}$ only the arterial-alveolar oxygen gradient was significant. With regard to other variables, $\mathrm{FEV}_{1}$ was significant in 5 studies, $5,9,11,12,27$ body mass index (BMI) was significant in 2 studies, $, 9,27$ and 
Peak Oxygen Uptake and Mortality in CF

Table 1. Main Methodological Characteristics of the Studies

\begin{tabular}{|c|c|c|c|c|c|}
\hline Study & Country of Origin & Type of Cohort & Sample Size & Age, $y^{*}$ & Follow-up, y \\
\hline Hulzebos et $\mathrm{al}^{9}$ & Netherlands & Prospective & 127 & $12.7(11-14)$ & 7.5 \\
\hline Van de Weert-van Leeuwen et al ${ }^{10}$ & Netherlands & Prospective & 149 & $13.2(12-13)$ & 2.8 \\
\hline Nguyen et $\mathrm{al}^{27}$ & France & Retrospective & 51 & $30.2(16-67)$ & 3 \\
\hline Pianosi et $\mathrm{al}^{12}$ & Canada & Prospective & 28 & $10(7-16)$ & $8 \dagger$ \\
\hline Moorcroft et al ${ }^{5}$ & United Kingdom & Retrospective & 87 & $19.8(15-40)$ & 5 \\
\hline Nixon et al ${ }^{11}$ & United States & Prospective & 109 & $17(7-35)$ & 8 \\
\hline
\end{tabular}

Table 2. Main Results of Cardiopulmonary Exercise Test Variables of the Studies Included in the Systematic Review

\begin{tabular}{|c|c|c|c|c|}
\hline Study & Baseline $\dot{\mathrm{V}}_{\mathrm{O}_{2}}$ Peak & Breathing Reserve at Maximum Exercise & $\dot{\mathrm{V}}_{\mathrm{E}} / \dot{\mathrm{V}}_{\mathrm{O}_{2}}$ & $\dot{\mathrm{V}}_{\mathrm{E}} / \dot{\mathrm{V}}_{\mathrm{CO}_{2}}$ \\
\hline Hulzebos et $\mathrm{al}^{9}$ & $\begin{array}{l}41.5 \pm 8.8 \mathrm{~mL} / \mathrm{kg} / \mathrm{min} \\
93.3 \pm 17.9 \% \text { predicted }\end{array}$ & $0.25 \pm 0.1^{*}$ & $37.4 \pm 6.8$ & $32.3 \pm 5.4$ \\
\hline Van de Weert-van Leeuwen et al ${ }^{10}$ & $\begin{array}{l}41.4 \pm 8.8 \mathrm{~mL} / \mathrm{kg} / \mathrm{min} \\
96.2 \pm 18.2 \% \text { predicted }\end{array}$ & NR & NR & NR \\
\hline Nguyen et al 27 & $\begin{array}{l}\text { Died: } 51.8 \pm 15.6 \% \text { predicted } \\
\text { Survived: } 66.1 \pm 20.7 \% \text { predicted }\end{array}$ & $\begin{array}{r}100.1 \pm 20.5 \dagger \\
74.5 \pm 21.3 \dagger\end{array}$ & NR & NR \\
\hline Pianosi et al ${ }^{12}$ & $41.2 \mathrm{~mL} / \mathrm{kg} / \mathrm{min}$ & $0.92 \div$ & NR & NR \\
\hline Moorcroft et al ${ }^{5}$ & $\begin{array}{l}\text { Died: } 53.7 \% \text { predicted } \\
\text { Survived: } 66.6 \% \text { predicted }\end{array}$ & NR & $\begin{array}{l}\text { Died: } 38.7 \\
\text { Survived: } 32.4\end{array}$ & NR \\
\hline Nixon et a $1^{11}$ & $\begin{array}{l}35 \mathrm{~mL} / \mathrm{kg} / \mathrm{min} \\
70 \% \text { predicted }\end{array}$ & NR & NR & NR \\
\hline $\begin{array}{l}* 1-(\text { Peak minute ventilation }- \text { maximum v } \\
\dagger \text { Peak minute ventilation }(\%) \text { of maximum vc } \\
¥ \text { Peak minute ventilation/maximum voluntary } \\
\dot{\mathrm{V}}_{\mathrm{O}_{2}}=\text { oxygen uptake } \\
\mathrm{NR}=\text { not reported } \\
\dot{\mathrm{V}}_{\mathrm{E}} / \mathrm{V}_{\mathrm{O}_{2}}=\text { ventilatory equivalent for oxygen } \\
\dot{\mathrm{V}}_{\mathrm{E}} / \dot{\mathrm{V}}_{\mathrm{CO}_{2}}=\text { ventilatory equivalent for carbon }\end{array}$ & $\begin{array}{l}\text { ntary ventilation). } \\
\text { tary ventilation. } \\
\text { ntilation. } \\
\text { kide }\end{array}$ & & & \\
\hline
\end{tabular}

Pseudomonas cepacia colonization was significant in 1 study. ${ }^{11}$ To determine the influence of CPET variables


Cox proportional hazards, whereas the other 3 studies used log rank tests, ${ }^{10}$ multivariate logistic regression, ${ }^{27}$ and chisquare tests. ${ }^{5}$

\section{Meta-analysis}

Two different analyses were carried out to evaluate the influence of $\dot{\mathrm{V}}_{\mathrm{O}_{2}}$ peak on mortality in subjects with $\mathrm{CF}$, and 2 studies were included in each analysis. Figure 2A shows data from articles reporting differences between averages of $\dot{\mathrm{V}}_{\mathrm{O}_{2}}$ peak in the survival and non-survival groups. The $\mathrm{I}^{2}$ analysis did not show heterogeneity between studies $\left(\mathrm{I}^{2}=0 \%, P=.84\right)$. The total difference standardized mean was -0.606 (CI 95\% $=-0.993$ to $-0.219, P=.002$ ), indicating that subjects in the nonsurvival group had a lower $\dot{\mathrm{V}}_{\mathrm{O}_{2}}$ peak compared to survivors.
Subjects with a lower $\dot{\mathrm{V}}_{\mathrm{O}_{2}}$ peak $(<45 \mathrm{~mL} / \mathrm{kg} / \mathrm{min}$ or $<82 \%$ of predicted) were associated with a significantly higher risk for mortality (relative risk 4.896, $95 \%$ CI $=1.086$ to $22.072, P=.039$ ). There was a small but not significant heterogeneity in the studies $\left(\mathrm{I}^{2}=31.56 \%, P=.23\right)$ included in this analysis (Fig. 2B). It was not possible to generate funnel charts in the analysis because of the small number of studies included.

\section{Discussion}

Low aerobic fitness has been reported in individuals with $\mathrm{CF}$ and is associated with several factors, including impairment of lung function, ${ }^{28}$ poor nutritional status, ${ }^{29}$ low muscle power, ${ }^{28}$ cardiac dysfunction, ${ }^{30}$ high level of inflammation, ${ }^{10}$ and physical deconditioning. ${ }^{28} \dot{\mathrm{V}}_{\mathrm{O}_{2}}$ peak, defined as the peak amount of oxygen that can be transferred and utilized during exercise, is the main parameter in the evaluation of aerobic capacity. ${ }^{18}$ Our systematic review and meta-analyses demonstrate that subjects with 


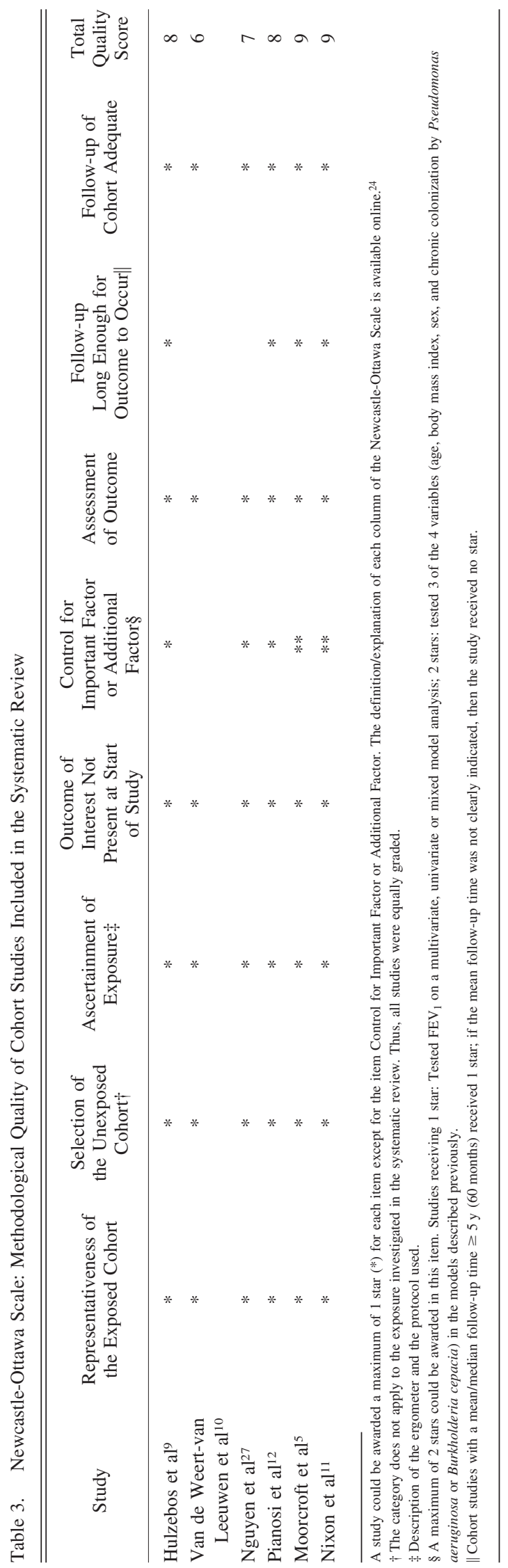

lower $\dot{\mathrm{V}}_{\mathrm{O}_{2}}$ peak rates (ie, values $<82 \%$ of predicted or $45 \mathrm{~mL} / \mathrm{kg} / \mathrm{min}$ ) demonstrated a 4.9 -fold increased risk for a fatal outcome, indicating that measurement of aerobic fitness could be a tool for prognosis in patients with CF. Furthermore, the difference between means indicated that subjects in the non-survival group had a lower $\dot{\mathrm{V}}_{\mathrm{O}_{2}}$ peak compared to the survivors. This association of oxygen consumption with mortality has also been described for individuals with $\mathrm{COPD}^{31,32}$ and chronic heart failure. ${ }^{33,34}$ To our knowledge, this is the first meta-analysis to evaluate the association of $\dot{\mathrm{V}}_{\mathrm{O}_{2}}$ peak with mortality in subjects with CF.

Nguyen et $\mathrm{al}^{27}$ was the only study included that found no relationship between $\dot{\mathrm{V}}_{\mathrm{O}_{2}}$ peak and mortality. They demonstrated only that alveolar-arterial gradient for oxygen at peak exercise was significantly associated with mortality. On the other hand, the remaining articles demonstrated an association between $\dot{\mathrm{V}}_{\mathrm{O}_{2}}$ peak and mortality. However, other variables, including $\mathrm{FEV}_{1}$ and $\mathrm{BMI}$, were also significant in predicting mortality. A higher BMI has been associated with an increased likelihood of survival in subjects with $\mathrm{CF},{ }^{7-9,35}$ corroborating the results from Nguyen et $\mathrm{al}^{27}$ and Hulzebos et al. ${ }^{9}$ Furthermore, FEV ${ }_{1}$ has been considered the best predictor of mortality in subjects with $\mathrm{CF}^{4,11,12}$ including those on lung transplant lists. ${ }^{36} \mathrm{Ac}-$ cording to Kerem et al ${ }^{4}$ individuals with $\mathrm{FEV}_{1}<30 \%$ of predicted should be referred for lung transplantation. Moorcroft et al ${ }^{5}$ showed that, despite the correlation of $\dot{V}_{\mathrm{O}_{2}}$ peak with survival, $\mathrm{FEV}_{1}$ is still the best indicator of prognosis. On the other hand, Rosenthal ${ }^{37}$ described that lung function between $8 \mathrm{y}$ and $12 \mathrm{y}$ was a poor indicator of the risk of dying or being transplanted. Moreover, Pianosi et al ${ }^{12}$ demonstrated that the change in $\dot{\mathrm{V}}_{\mathrm{O}_{2}}$ peak over time is more useful as a prognostic marker than the commonly used longitudinal decline in $\mathrm{FEV}_{1}$. In addition, abnormalities in aerobic fitness in early disease in stable subjects may reflect changes that are not detected with spirometry, given that Dodd et al ${ }^{38}$ have demonstrated that the correlation between thoracic computed tomography abnormalities and exercise limitation is stronger than the correlation between spirometry and BMI with exercise limitation. Thus, it is possible that $\dot{\mathrm{V}}_{\mathrm{O}_{2}}$ peak is a better prognostic marker early in life than $\mathrm{FEV}_{1}$, considering that CPET is dependent on more than lung function and thus reflects the effects of the disease on other organ systems earlier on; however, further studies are needed to directly address this question.

Information obtained through CPET plays an important role in the care and follow-up of patients with $\mathrm{CF}$ because of its contribution to prognosis and functional information. ${ }^{18}$ Thus, an annual follow-up of $\dot{\mathrm{V}}_{\mathrm{O}_{2}}$ peak is relevant to identify individuals at risk for a worsening prognosis, ${ }^{15}$ as well as those who may benefit from more intense therapy, ${ }^{39}$ considering that aerobic fitness correlates with sur- 


\section{Peak Oxygen Uptake and Mortality in CF}

Table 4. Variables Used as Mortality Predictors in Patients with Cystic Fibrosis

\begin{tabular}{|c|c|c|}
\hline Study & Exercise Capacity Variables & Other Variables \\
\hline Hulzebos et $\mathrm{al}^{9}$ & $\begin{array}{l}\dot{\mathrm{V}}_{\mathrm{O}_{2}} / \mathrm{kg}(\% \text { predicted }): 2.96(1.06-8.23)^{*} \\
\text { Breathing reserve: } 3.35(1.19-9.48)^{*}\end{array}$ & $\begin{array}{l}\mathrm{FEV}_{1} \%: 17.84(4.02-79.08)^{*} \\
\text { BMI: } 8.1(2.93-22.51)^{*}\end{array}$ \\
\hline $\begin{array}{l}\text { Van de Weert-van } \\
\text { Leeuwen et al }{ }^{10}\end{array}$ & $\begin{array}{l}\text { Maximum } \dot{\mathrm{V}}_{\mathrm{O}_{2}} / \mathrm{kg}(\% \text { predicted }) \leq 80: 96.3 \% \text { mortality } \dagger \\
\text { Maximum } \dot{\mathrm{V}}_{\mathrm{O}_{2}} / \mathrm{kg}(\% \text { predicted })>80: 80.0 \% \text { mortality }\end{array}$ & NA \\
\hline Nguyen et $\mathrm{al}^{27}$ & Peak $\mathrm{P}_{(\mathrm{A}-\mathrm{a}) \mathrm{O}_{2}}: 0.794(0.668-0.943, P=.009) \ddagger$ & $\begin{array}{l}\mathrm{FEV}_{1} \%: 1.015(0.904-1.139, P=.80) \ddagger \\
\text { Body mass index: } 1.8(1.1-2.9, P=.03) \ddagger\end{array}$ \\
\hline Pianosi et al ${ }^{12}$ & $\begin{array}{l}\text { Peak } \dot{\mathrm{V}}_{\mathrm{O}_{2}} \text { intercept: } 0.910(0.816-1.014, P=.09)^{*} \\
\text { Peak } \dot{\mathrm{V}}_{\mathrm{O}_{2}} \text { slope: } 0.048(0.005-0.430, P=.007)^{*}\end{array}$ & $\begin{array}{l}\text { FEV }_{1} \text { intercept: } 0.915(0.868-0.964, P<.001)^{*} \\
\text { FEV }_{1} \text { slope: } 0.008(0.0003-0.210, P=.004)^{*}\end{array}$ \\
\hline Moorcroft et $\mathrm{al}^{5}$ & $\begin{array}{l}\text { Peak } \dot{\mathrm{V}}_{\mathrm{O}_{2}} \text { (cut-off } 56 \% \text { ): } 64 \% \text { sensitivity and } \\
72 \% \text { specificity }\end{array}$ & $\mathrm{FEV}_{1}$ (cut-off $55 \%$ ): $91 \%$ sensitivity and $74 \%$ specificity \\
\hline Nixon et a $1^{11}$ & Peak $\dot{\mathrm{V}}_{\mathrm{O}_{2}} \%(\leq 58$ vs $\geq 82): 3.2(1.2-8.6, P=.02) \S$ & $\begin{array}{l}\mathrm{FEV}_{1} \%(\leq 50 \text { vs } \geq 65): 1.1(0.4-2.7, P=.83) \S \\
\text { Pseudomonas cepacia }(\text { present vs absent }): \\
\quad 5.0(2.6-9.5, P<.001) \S\end{array}$ \\
\hline $\begin{array}{l}\text { * Hazard ratio }(95 \% \mathrm{CI}) \text {. } \\
\dagger P=.02 . \\
\text { † Odds ratio }(95 \% \mathrm{CI}) \text {. } \\
\text { § Relative risk }(95 \% \mathrm{CI}) \text {. } \\
\dot{\mathrm{V}}_{\mathrm{O}_{2}}=\text { oxygen uptake } \\
\mathrm{P}_{(\mathrm{A}-\mathrm{a}) \mathrm{O}_{2}}=\text { alveolar-arteria } \\
\mathrm{BMI}=\text { body mass index }\end{array}$ & ifference & \\
\hline
\end{tabular}



Fig. 2. Meta-analysis of studies that reported mean difference in $\dot{\mathrm{V}}_{\mathrm{O}_{2}}$ peak between survival or non-survival groups (A) and the association (relative risk, $95 \% \mathrm{Cl}$ ) of $\dot{\mathrm{V}}_{\mathrm{O}_{2}}$ peak (high vs. low-moderate) with mortality (B). Low $\dot{\mathrm{O}}_{\mathrm{O}_{2}}$ peak was considered when $<45 \mathrm{~mL} / \mathrm{kg} / \mathrm{min}$ or $82 \%$ of predicted.

vival in children ${ }^{12}$ and adults ${ }^{5,11}$ with CF. In addition, it is well-established that exercise increases $\dot{\mathrm{V}}_{\mathrm{O}_{2}}$ peak, indicating its importance as a therapeutic tool that could influence prognosis. ${ }^{40}$

The studies included in this systematic review and metaanalysis showed variation in follow-up time, ranging from $2.8 \mathrm{y}$ to $8 \mathrm{y}$. Considering that these are studies to evaluate mortality, a short follow-up time may be considered as a limitation because it could influence this outcome. In addition, it is already known that the evolution of $\mathrm{CF}$ is characterized by a decline in lung function and exercise limitation, ${ }^{1}$ highlighting the role of long-term follow-up periods in the evaluation of mortality rates.

As for the reported variables of CPET, the included studies showed a large variability, which prevented us from including some articles in certain analyses and to evaluate the influence of other variables such as ventilatory reserve data, $\dot{\mathrm{V}}_{\mathrm{E}} / \dot{\mathrm{V}}_{\mathrm{O}_{2}}$, and $\dot{\mathrm{V}}_{\mathrm{E}} / \dot{\mathrm{V}}_{\mathrm{CO}_{2}}$. A high breathing reserve index at the lactate threshold represents a reduction of pulmonary mechanical reserve and was already reported as a predictor of mortality in individuals with $\mathrm{CF}$ waiting for lung transplantation. ${ }^{25}$ In addition, $\dot{\mathrm{V}}_{\mathrm{E}} / \dot{\mathrm{V}}_{\mathrm{O}_{2}}$ mea- 


\section{Peak Oxygen Uptake and Mortality in CF}

surement has also been shown to be important in predicting mortality. ${ }^{5,9}$ Despite the relevance of the analyzed variables, it is important to recognize that CPET is not widely available in $\mathrm{CF}$ centers and requires specific equipment and expert personnel to perform and interpret the test. On the other hand, increasing life expectancy and new therapeutic options are changing the clinical presentation of $\mathrm{CF}$, and markers such as lung function are better maintained over time, highlighting the importance of other factors such as aerobic fitness. Thus, studies designed to evaluate the prognostic value of CPET as compared to nutritional status, lung function, and other exercise tests are needed to further understand the role of aerobic fitness as a disease progression marker in CF.

Regarding the methodological quality of the studies included, in general, a high quality was found because only 1 study ${ }^{10}$ presented a reasonable classification according to the Newcastle-Ottawa Scale, which strengthens our systematic review and meta-analysis. In addition, the $\mathrm{I}^{2}$ test in the analysis of the difference between means did not show heterogeneity, and the relative risk analysis showed a low heterogeneity among the included studies, which may confirm the power of the analysis presented, despite the small number of articles.

One of the main limitations of this study is the small number of articles included in each analysis and the variability of the parameters evaluated. Due to the different presentations of data, only 2 articles were included in each of the analyses. Therefore, it was not possible to perform a funnel chart to evaluate publication bias of the studies. A short follow-up period, especially for a population of young children and adolescents, may also be considered a limitation in the evaluation of mortality. Two studies 9,10 included subjects from the same CF center and with similar characteristics, which may include some shared members of a cohort. In addition, the lack of response when we tried to contact the authors of some of the studies ${ }^{9-12}$ to request additional $\dot{\mathrm{V}}_{\mathrm{O}_{2}}$ peak information distinguished between survivors and non-survivors may be considered a limitation of present study.

\section{Conclusions}

This systematic review and meta-analysis showed that a low level of peak oxygen uptake is associated with higher mortality in $\mathrm{CF}$, as subjects with reduced $\dot{\mathrm{V}}_{\mathrm{O}_{2}}$ peak had a 4.9-fold increased risk for a fatal outcome. This indicates that $\dot{\mathrm{V}}_{\mathrm{O}_{2}}$ peak may be an important follow-up variable to measure in addition to $\mathrm{FEV}_{1}$, although data from our analyses should be interpreted with caution due to the small number of studies included. Moreover, comparisons between aerobic capacity variables, $\mathrm{FEV}_{1}$, and $\mathrm{BMI}$ as mortality predictors in $\mathrm{CF}$ need to be further explored in future studies.

\section{REFERENCES}

1. Gibson RL, Burns JL, Ramsey BW. Pathophysiology and management of pulmonary infections in cystic fibrosis. Am J Respir Crit Care Med 2003;168(8):918-951.

2. Cystic Fibrosis Foundation Patient Registry, 2015 Annual Data Report. Available at https://www.cff.org/Our-Research/CF-PatientRegistry/2015-Patient-Registry-Annual-Data-Report.pdf Accessed Feb 20, 2017.

3. Currie S, Greenwood K, Weber L, Khakee H, Legasto M, Tullis E, et al. Physical activity levels in individuals with cystic fibrosisrelated diabetes. Physiother Can 2017;69(2):171-177.

4. Kerem E, Reisman J, Corey M, Canny GJ, Levison H. Prediction of mortality in patients with cystic fibrosis. N Engl J Med 1992;326(18): 1187-1191.

5. Moorcroft AJ, Dodd ME, Webb AK. Exercise testing and prognosis in adult cystic fibrosis. Thorax 1997;52(3):291-293.

6. Buzzetti R, Alicandro G, Minicucci L, Notarnicola S, Furnari ML, Giordano G, et al. Validation of a predictive survival model in Italian patients with cystic fibrosis. J Cyst Fibros 2012;11(1):24-29.

7. Liou TG, Adler FR, FitzSimmons SC, Cahill BC, Hibbs JR, Marshall BC. Predictive 5-year survivorship model of cystic fibrosis. Am J Epidemiol 2001;153(4):345-352

8. Fogarty AW, Britton J, Clayton A, Smyth AR. Are measures of body habitus associated with mortality in cystic fibrosis? Chest 2012; 142(3):712-717.

9. Hulzebos EH, Bomhof-Roordink H, van de Weert-van Leeuwen PB, Twisk JW, Arets HG, van der Ent CK, Takken T. Prediction of mortality in adolescents with cystic fibrosis. Med Sci Sports Exerc 2014;46(11):2047-2052.

10. Van de Weert-van Leeuwen P, Slieker M, Hulzebos H, Kruitwagen $\mathrm{C}$, van der Ent C, Arets H. Chronic infection and inflammation affect exercise capacity in cystic fibrosis. Eur Respir J 2012;39(4):893-898.

11. Nixon PA, Orenstein DM, Kelsey SF, Doershuk CF. The prognostic value of exercise testing in patients with cystic fibrosis. N Engl J Med 1992;327(25):1785-1788.

12. Pianosi P, Leblanc J, Almudevar A. Peak oxygen uptake and mortality in children with cystic fibrosis. Thorax 2005;60(1):50-54.

13. Troosters T, Langer D, Vrijsen B, Segers J, Wouters K, Janssens W, et al. Skeletal muscle weakness, exercise tolerance and physical activity in adults with cystic fibrosis. Eur Respir J 2009;33(1):99106.

14. American Thoracic Society. ATS/ACCP statement on cardiopulmonary exercise testing. Am J Respir Crit Care Med 2003;167(2):211217.

15. Hebestreit H, Arets HG, Aurora P, Boas S, Cerny F, Hulzebos EH, et al. Statement on exercise testing in cystic fibrosis. Respiration 2015;90(4):332-351.

16. Pianosi P, LeBlanc J, Almudevar A. Relationship between $\mathrm{FEV}_{1}$ and peak oxygen uptake in children with cystic fibrosis. Pediatr Pulmonol 2005;40(4):324-329.

17. Savi D, Di Paolo M, Simmonds N, Onorati P, Internullo M, Quattrucci $\mathrm{S}$, et al. Relationship between daily physical activity and aerobic fitness in adults with cystic fibrosis. BMC Pulm Med 2015;15(1):59.

18. Ferrazza A, Martolini D, Valli G, Palange P. Cardiopulmonary exercise testing in the functional and prognostic evaluation of patients with pulmonary diseases. Respiration 2009;77(1):3-17.

19. Saynor ZL, Barker AR, Oades PJ, Williams CA. Reproducibility of maximal cardiopulmonary exercise testing for young cystic fibrosis patients. J Cyst Fibros 2013;12(6):644-650.

20. McKone E, Barry S, FitzGerald M, Gallagher C. Reproducibility of maximal exercise ergometer testing in patients with cystic fibrosis. Chest 1999;116(2):363-368.

21. De Jong W, Van Der Schans C, Mannes G, Van Aalderen W, Grevink R, Koeter G. Relationship between dyspnoea, pulmonry function and 


\section{Peak Oxygen Uptake and Mortality in CF}

exercise capacity in patients with cystic fibrosis. Respir Med 1997; 91(1):41-46.

22. Stroup DF, Berlin JA, Morton SC, Olkin I, Williamson GD, Rennie D, et al. Meta-analysis of observational studies in epidemiology: a proposal for reporting. JAMA 2000;283(15):2008-2012.

23. Liberati A, Altman DG, Tetzlaff J, Mulrow C, Gøtzsche PC, Ioannidis JP, et al. The PRISMA statement for reporting systematic reviews and meta-analyses of studies that evaluate health care interventions: explanation and elaboration. Ann Intern Med 2009;151(4):65-94.

24. Wells G, Shea B, O'Connell D, Peterson J, Welch V, Losos M, et al. The Newcastle-Ottawa Scale (NOS) for assessing the quality of nonrandomised studies in meta-analyses. Available at http//www.ohri.ca/ programs/clinical_epidemiology/oxford.asp Accessed Mar 27, 2017.

25. Tantisira KG, Systrom DM, Ginns LC. An elevated breathing reserve index at the lactate threshold is a predictor of mortality in patients with cystic fibrosis awaiting lung transplantation. Am J Respir Crit Care Med 2002;165(12):1629-1633.

26. Stanchina ML, Tantisira KG, Aquino SL, Wain JC, Ginns LC. Association of lung perfusion disparity and mortality in patients with cystic fibrosis awaiting lung transplantation. J Heart Lung Transplant 2002;21(2):217-225.

27. Nguyen S, Leroy S, Cracowski C, Perez T, Valette M, Neviere R, et al. [Prognostic value of clinical exercise testing in adult patients with cystic fibrosis]. Rev Mal Respir 2010;27(3):219-225.

28. Hebestreit H, Kieser S, Rüdiger S, Schenk T, Junge S, Hebestreit A, et al. Physical activity is independently related to aerobic capacity in cystic fibrosis. Eur Respir J 2006;28(4):734-739.

29. Klijn PH, van der Net J, Kimpen JL, Helders PJ, Van der Ent CK. Longitudinal determinants of peak aerobic performance in children with cystic fibrosis. Chest 2003;124(6):2215-2219.

30. Pianosi P, Pelech A. Stroke volume during exercise in cystic fibrosis. Am J Respir Crit Care Med 1996;153(3):1105-1109.

31. Oga T, Nishimura K, Tsukino M, Sato S, Hajiro T. Analysis of the factors related to mortality in chronic obstructive pulmonary disease: role of exercise capacity and health status. Am J Respir Crit Care Med 2003;167(4):544-549.

32. Celli BR. Predictors of mortality in COPD. Respir Med 2010;104(6): 773-779.

33. Ingle L, Witte KK, Cleland JG, Clark AL. Combining the ventilatory response to exercise and peak oxygen consumption is no better than peak oxygen consumption alone in predicting mortality in chronic heart failure. Eur J Heart Fail 2008;10(1):85-88.

34. Cicoira M, Davos CH, Francis DP, Doehner W, Zanolla L, Franceschini L, et al. Prediction of mortality in chronic heart failure from peak oxygen consumption adjusted for either body weight or lean tissue. J Card Fail 2004;10(5):421-426.

35. Simmonds NJ, MacNeill SJ, Cullinan P, Hodson ME. Cystic fibrosis and survival to 40 years: a case-control study. Eur Respir J 2010; 36(6):1277-1283

36. Belkin RA, Henig NR, Singer LG, Chaparro C, Rubenstein RC, Xie SX, et al. Risk factors for death of patients with cystic fibrosis awaiting lung transplantation. Am J Respir Crit Care Med 2006; 173(6):659-666.

37. Rosenthal M. Annual assessment spirometry, plethysmography, and gas transfer in cystic fibrosis: do they predict death or transplantation. Pediatr Pulmonol 2008;43(10):945-952.

38. Dodd JD, Barry SC, Barry RB, Gallagher CG, Skehan SJ, Masterson JB. Thin-section CT in patients with cystic fibrosis: correlation with peak exercise capacity and body mass index 1. Radiology 2006; 240(1):236-245.

39. Javadpour S, Selvadurai H, Wilkes D, Schneiderman-Walker J, Coates A. Does carbon dioxide retention during exercise predict a more rapid decline in $\mathrm{FEV}_{1}$ in cystic fibrosis? Arch Dis Child 2005;90(8): 792-795.

40. Sosa ES, Groeneveld IF, Gonzalez-Saiz L, Lopez-Mojares LM, Villa-Asensi JR, Gonzalez MIB, et al. Intrahospital weight and aerobic training in children with cystic fibrosis: a randomized controlled trial. Med Sci Sports Exerc 2012;44(1):2-11. 